\title{
Anti-PEG antibodies alter the mobility and biodistribution of densely PEGylated nanoparticles in mucus
}

Christine E. Henry ${ }^{b}$, Ying-Ying Wang ${ }^{d}$, Qi Yang ${ }^{a}$, Thuy Hoang ${ }^{\mathrm{e}}$, Sumon Chattopadhyay ${ }^{\mathrm{f}}$, Timothy Hoen ${ }^{\mathrm{d}}$, Laura M. Ensign ${ }^{\mathrm{f}, \mathrm{g}}$, Kenetta L. Nunn ${ }^{\mathrm{b}}$, Holly Schroeder ${ }^{\mathrm{a}}$, Justin McCallen ${ }^{\mathrm{a}}$, Thomas Moench ${ }^{\mathrm{h}}$, Richard Cone $^{d}$, Steve R. Roffleri, Samuel K. Lai ${ }^{\mathrm{a}-\mathrm{c}, *}$

${ }^{a}$ Division of Molecular Pharmaceutics, Eshelman School of Pharmacy; ${ }^{b}$ UNC/NCSU Joint Department of Biomedical Engineering; ${ }^{C}$ Department of Microbiology \& Immunology, University of North Carolina Chapel Hill, 125 Mason Farm Road, Chapel Hill, NC 27599, USA.

' Department of Biophysics, Johns Hopkins University, 3400 North Charles Street, Baltimore, MD 21218, USA.

${ }^{\mathrm{e}}$ Department of Pharmacology and Molecular Sciences; ${ }^{\mathrm{f}}$ Center for Nanomedicine; ${ }^{\mathrm{g}}$ Department of Ophthalmology, Johns Hopkins University School of Medicine, 600 N Wolfe St, Baltimore, MD 21287, USA.

${ }^{\mathrm{h}}$ ReProtect, Inc., 703 Stags Head Rd, Baltimore, MD 21286, USA.

' Institute of Biomedical Sciences, Academia Sinica, No. 128, Section 2, Academia Road, Taipei 11529, Taiwan.

* Corresponding author: lai@unc.edu

Division of Molecular Pharmaceutics

University of North Carolina at Chapel Hill

Marsico Hall 4213, 125 Mason Farm Road

Campus Box 7362

Chapel Hill, NC 27599-7360

T. (919) 966-3024

\begin{abstract}
Antibodies that specifically bind polyethylene glycol (PEG) can lead to rapid elimination of PEGylated therapeutics from the systemic circulation. We have recently shown that virus-binding IgG can immobilize viruses in mucus via multiple low-affinity crosslinks between IgG and mucins. However, it remains unclear whether anti-PEG antibodies in mucus may also alter the penetration and consequently biodistribution of PEGylated nanoparticles delivered to mucosal surfaces. We found that both anti-PEG IgG and IgM can readily bind nanoparticles that were densely coated with PEG polymer to minimize adhesive interactions with mucus constituents. Addition of anti-PEG IgG and IgM into mouse cervicovaginal mucus resulted in extensive trapping of mucus-penetrating PEGylated nanoparticles, with the fraction of mobile particles reduced from over $95 \%$ to only $34 \%$ and $7 \%$ with anti-PEG IgG and IgM, respectively. Surprisingly, we did not observe significant agglutination induced by either antibody, suggesting that particle immobilization is caused by adhesive crosslinks between mucin fibers and IgG or IgM bound to individual nanoparticles. Importantly, addition of corresponding control antibodies did not slow the PEGylated nanoparticles, confirming anti-PEG antibodies specifically bound to and trapped the PEGylated nanoparticles. Finally, we showed that trapped PEGylated nanoparticles remained largely in the luminal mucus layer of the mouse vagina even when delivered in hypotonic formulations that caused untrapped particles to be drawn by the flow of water (advection) through mucus all the way to the epithelial surface. These results underscore the potential importance of elucidating mucosal antiPEG immune responses for PEGylated therapeutics and biomaterials applied to mucosal surfaces.
\end{abstract}

Keywords: PEGylation; IgG; IgM; mucosal immunity; nanomedicine; diffusion 


\section{Introduction}

All exposed surfaces in the human body not covered by skin, including those of the respiratory, gastrointestinal and urogenital tracts, are covered with a layer of mucus secretions. In addition to mucins secreted by goblet cells and mucin-secreting glands, mucus also contains various proteins, lipids, cells and other constituents, all of which contribute to the proper functioning of mucus as a lubricant and diffusional barrier against foreign particulates [1]. Mucus is continuously secreted and cleared, which enables it to function as a constantly renewed filter that can rapidly eliminate any foreign particulates trapped within the dense network of mucin fibers [2]. To overcome the mucus barrier, we and others have engineered nanoparticles with minimal affinity to mucus constituents [3-6], which can undergo rapid diffusion in the low viscosity interstitial fluids between mucin fibers [7] as well as transport through the mucus gel by advective bulk fluid flow [8]. The most popular approach to formulate such mucus-penetrating particles has been to coat particles with a dense layer of polyethylene glycol (PEG) [3, 4, 9]; the resulting 'mucus-penetrating particles' have been shown to markedly improve the delivery of chemotherapeutics against cervical cancer [10], microbicides against vaginal Herpes transmission [11], and DNA in the lung airways [12]. These successes have expanded the use of PEGylation, which is already routinely employed to prolong the circulation kinetics of proteins, liposomes and polymeric nanoparticles [13-16], to mucosal applications.

The increasing use of PEG in drug delivery applications, including those that require repeat dosing, has led to investigations of potential immune responses upon repeated or prolonged exposure to PEGylated therapeutics. Since the late 1990s, several groups have reported that a second dose of PEG-modified liposomes is generally rapidly cleared from the blood and accumulates in the liver when injected into the same rat or mouse at several-day intervals $[17,18]$. This accelerated blood clearance phenomenon, which results in markedly reduced efficacy for some PEGylated therapeutics, was later found to be mediated by induced antibodies that specifically bind PEG [19-21]. Schellekens et al. [22] recently raised considerable concerns regarding the anti-PEG antibody phenomena by summarizing some of the variations and apparent contradictions across different studies, and astutely highlighted the lack of validated and standardized assays as a major barrier in correctly characterizing anti-PEG antibody responses in biological specimens. Nevertheless, given the sheer volume of observations for anti-PEG antibody response by numerous independent investigators across different animal models and different PEGylated systems, it is perhaps more likely that the variable observations reflect an incomplete understanding of the complexity of anti-PEG phenomena in vivo rather than solely inadequately supported conclusions based on artifacts from ELISA and other assays.

It is important to also note that all in vivo studies in the literature were performed in animals that had no prior exposure to PEG. In contrast, humans are increasingly exposed to PEG found in everyday products including detergents, food products and cosmetics. Due to this repeated exposure, a substantial proportion of the human population likely has pre-existing anti-PEG antibodies. In 2003, Armstrong et al. reported anti-PEG IgG and IgM in $22.5 \%$ and $8.6 \%$ of serum samples, respectively [23], and those proportions have likely increased over the past decade. Indeed, pilot studies in our lab suggest pre-existing anti-PEG immunity may be even more prevalent [24]. Possible flaws with detection assays [22] notwithstanding, these findings clearly underscore the importance of evaluating the influence of pre-existing anti-PEG immunity in any human studies of PEGylated therapeutics. Furthermore, because PEG exposure may occur at mucus membranes (e.g., via oral exposure to PEG in dental care products, gastrointestinal exposure to PEG in food additives, or vaginal exposure to PEG in personal lubricants or vaginal creams), mucosal anti-PEG responses capable of altering the biodistribution and pharmacokinetics of topically applied PEGylated carriers are a distinct possibility.

An emerging mechanism of mucosal immune protection, pioneered by our group, is the immobilization of individual viruses due to antibody-mucin interactions, which in turn prevents viral translocation across mucus [25]. Previous work has shown that the interactions between antibodies and 
mucus are low-affinity and transient; for example, the diffusion of IgG and IgA molecules (diameter $~ 10$ $\mathrm{nm}$ ) in human mucus (pores $340 \pm 70 \mathrm{~nm}$ [7]) is slowed only 5\%-20\% compared to in buffer [26, 27]. We found that this seemingly negligible affinity is sufficient to trap HSV-1 virions in human cervicovaginal mucus (CVM) with sub-neutralizing potency, presumably because the array of virionbound IgG ensures a sufficient number of transient low-affinity bonds between the virus/IgG complex and mucins at any given time [25]. Furthermore, a non-neutralizing IgG facilitated effective protection against vaginal Herpes infection in mice, but that protection was abolished when vaginal mucus was removed. These results indicate that virus-binding antibodies in mucus can directly alter the mobility of virions in mucus, leading to a markedly reduced flux arriving at the epithelium [27]. We thus sought to evaluate here whether anti-PEG antibodies in mucus would similarly trap PEGylated nanoparticles in mucus and impede therapeutics delivery to mucosal surfaces.

\section{Methods}

\subsection{Mouse cervicovaginal mucus collection}

Mouse cervicovaginal mucus (mCVM) was obtained from 6-8 week old CF-1 mice (Harlan, Frederick, $\mathrm{MD}$ ), injected subcutaneously with $2.5 \mathrm{mg}$ of Depo-Provera ${ }^{\circledR}$ (DP; medroxyprogesterone acetate) (Pharmacia \& Upjohn Company, Kalamazoo, MI) seven days prior to experiments. mCVM was collected by lavage with $20 \mu \mathrm{L}$ of normal saline, and mCVM from 10-15 mice was pooled to collect sufficient quantities for multiple particle tracking studies. The collection procedure yields highly viscoelastic mucus, as observed visually and through particle tracking experiments. Mucus was stored at $4{ }^{\circ} \mathrm{C}$ until used for microscopy within $48 \mathrm{hrs}$. Mice were anesthetized prior to experimental procedures. All experimental protocols were approved by the University of North Carolina at Chapel Hill Institutional Animal Care and Use Committee.

\subsection{Nanoparticle preparation and characterization}

To produce PEGylated nanoparticles (PS-PEG), we covalently modified $100 \mathrm{~nm}$ fluorescent, carboxylmodified polystyrene beads (PS; Molecular Probes, Eugene, OR) with 2 kDa methoxy poly(ethylene glycol) amine (PEG; Rapp Polymere, Tuebingen, Germany) via a carboxyl-amine reaction, as published previously $[4,28]$. Particle size and $\zeta$-potential were determined by dynamic light scattering and laser Doppler anemometry, respectively, using a Zetasizer Nano ZS (Malvern Instruments, Southborough, MA). Size measurements were performed at $25^{\circ} \mathrm{C}$ at a scattering angle of $90^{\circ}$. Samples were diluted in $10 \mathrm{mM} \mathrm{NaCl}$ solution, and measurements were performed according to instrument instructions. High density PEGylation (>1 PEG/ $\mathrm{nm}^{2}$ ) was verified using the fluorogenic compound 1-pyrenyldiazomethane (PDAM) to quantify residual unmodified carboxyl groups on the polystyrene beads [28]. PEG conjugation was also confirmed by a near-neutral $\zeta$-potential (Table 1) [4].

\subsection{Anti-PEG IgG and IgM antibodies}

Mouse anti-PEG IgG (3.3) and IgM (AGP-4) antibodies are commercially available from Academia Sinica [29] and have been previously characterized [30]. The binding avidity for anti-PEG IgG (3.3) has been reported to be $1.3 \times 10^{-7} \mathrm{M}$ at room temperature [31], while that for anti-PEG IgM (AGP.4) is at least 1.4 $x 10^{-10} \mathrm{M}$ [32]. To confirm the specificity of the anti-PEG antibodies used in our studies, we performed a competitive ELISA. Medium binding EIA/RIA 96-well plates (Corning, Corning, NY) were coated with 50 $\mu \mathrm{g} / \mathrm{mL}$ of 1,2-distearoyl-sn-glycero-3-phosphoethanolamine- $\mathrm{N}$-[amino(polyethylene glycol)-5000] (DSPE$\mathrm{PEG}_{5 \mathrm{k}}$, Nanocs, New York, NY) in PBS overnight at $4^{\circ} \mathrm{C}$. Anti-PEG IgG or control IgG antibody was added at $6 \mu \mathrm{g} / \mathrm{mL}$, while anti-PEG IgM or control IgM antibody was added at $2 \mu \mathrm{g} / \mathrm{mL}$. The antibodies were incubated with the plate in the absence or presence of free diol $\mathrm{PEG}_{8 \mathrm{k}}$ (Sigma-Aldrich). Bound antibody 
was detected using goat anti-human IgG HRP (Santa Cruz, Biotechnology, Dallas, TX) or anti-human IgM HRP (Rockland Immunochemicals, Pottstown, PA) and then 1-step Ultra TMB substrate (Thermo Scientific, Waltham, MA), and absorbance at $450 \mathrm{~nm}$ was read using a Spectramax M2 (Molecular Devices, Sunnyvale, CA). No PEG-containing detergent was used in any of the ELISA assays.

Anti-PEG IgG and IgM binding to PS-PEG nanoparticles were confirmed by dot blot assay. Two microliters of PS or PS-PEG beads were blotted onto nitrocellulose membranes. The membranes were incubated with 1:2000 dilutions of anti-PEG IgM, control IgM (sc-58070 Vancomycin (2F10); Santa Cruz Biotechnology, Dallas, TX), anti-PEG IgG, or control IgG (sc-101339 Biotin (33); Santa Cruz Biotechnology, Dallas, TX), followed by incubation with a 1:10000 dilution of HRP-labeled goat anti-mouse IgM (Life Technologies, Carlsbad, CA) or a 1:7000 dilution of HRP-labeled goat anti-mouse IgG (Santa Cruz Biotechnology, Dallas, TX). Bound secondary antibody was detected using an ECL kit (BioRad, Hercules, CA), and imaged using a FluorChem E system (ProteinSimple, San Jose, CA). The same anti-PEG and control IgM were used in both multiple particle tracking and in vivo studies.

\subsection{Multiple particle tracking}

For particle tracking studies, anti-PEG or control IgG or IgM as listed above were first added to $20 \mu \mathrm{L}$ of mCVM in custom-made chambers, followed by addition of dilute particle solutions ( $10^{8}-10^{9}$ particles $/ \mathrm{mL}, 5 \% \mathrm{v} / \mathrm{v})$. To confirm the specificity of anti-PEG antibodies to PEG, free PEG ( $8 \mathrm{kDa}, 0.2$ $\mathrm{mg} / \mathrm{mL}$; Sigma, St. Louis, $\mathrm{MO}$ ) was also added to $\mathrm{mCVM}$ prior to the addition of particles. Samples were incubated $1 \mathrm{hr}$ at $37^{\circ} \mathrm{C}$ before microscopy. The trajectories of the fluorescent particles in $\mathrm{mCVM}$ were recorded using an EMCCD camera (Evolve 512; Photometrics, Tucson, AZ) mounted on an inverted epifluorescence microscope (AxioObserver D1; Zeiss, Thornwood, NY), equipped with an Alpha Plan-Apo 100x/1.46 NA objective, environmental (temperature and $\mathrm{CO}_{2}$ ) control chamber and an LED light source (Lumencor Light Engine DAPI/GFP/543/623/690). Videos (512 x 512, 16-bit image depth) were captured with MetaMorph imaging software (Molecular Devices, Sunnyvale, CA) at a temporal resolution of 66.7 $\mathrm{ms}$ and spatial resolution of $10 \mathrm{~nm}$ (nominal pixel resolution $0.156 \mu \mathrm{m} /$ pixel). The tracking resolution was determined by tracking the displacements of particles immobilized with a strong adhesive, following a previously described method [33]. Particle trajectories were analyzed using MATLAB software as described previously [34]. Sub-pixel tracking resolution was achieved by determining the precise location of the particle centroid by light-intensity-weighted averaging of neighboring pixels. Trajectories of $n \geq 40$ particles per frame on average (corresponding to $n \geq 100$ total traces) were analyzed for each experiment, and four independent experiments were performed in $\mathrm{mCVM}$ collected from different mice. The coordinates of particle centroids were transformed into time-averaged mean squared displacements (MSD), calculated as $\left\langle\Delta r^{2}(\tau)\right\rangle=[x(t+\tau)-x(t)]^{2}+[y(t+\tau)-y(t)]^{2}$ (where $\tau=$ time scale or time lag), from which distributions of MSDs and effective diffusivities ( $D_{\text {eff }}$ ) were calculated, as previously demonstrated $[4,35,36]$. MSD may also be expressed as $M S D=4 D_{0} \tau^{\alpha}$, where $\alpha$, the slope of the curve on a log-log scale, is a measure of the extent of impediment to particle diffusion ( $\alpha=1$ for pure unobstructed Brownian diffusion; $\alpha<1$ indicates increasing impediment to particle movement as $\alpha$ decreases). Mobile particles were defined as those with $D_{\text {eff }} \geq 10^{-1.5} \mu \mathrm{m}^{2} / \mathrm{s}$ at $\tau=0.2667 \mathrm{~s}$ (this $\tau$ corresponds to a minimum trajectory length of 5 frames), based on multiple datasets of mobile and immobile nanoparticles (e.g., PS and PS-PEG nanoparticles) in human mucus [7, 37]. For particles 100 $\mathrm{nm}$ in size, $a D_{\text {eff }} \geq 10^{-1.5} \mathrm{\mu m}^{2} / \mathrm{s}$ effectively means that the particles move a distance greater than their diameters within $0.2667 \mathrm{~s}$.

\subsection{Distribution of nanoparticles in the mouse vagina}

To evaluate PEGylated nanoparticle trapping in vivo, we used female 6-8 week old CF-1 mice (Harlan, Frederick, MD) pretreated with $100 \mathrm{mg}$ of $17 \beta$-estradiol benzoate (Sigma, St. Louis, MO) injected 
subcutaneously two days before the experiments [11]. Anti-PEG or control (anti-vancomycin) IgM was first administered to the mouse vagina in two doses: $10 \mu \mathrm{L}$ of $150 \mu \mathrm{g} / \mathrm{mL}$ antibody in $0.5 \mathrm{x}$ normal saline, and after a $10 \mathrm{~min}$ interval, another $10 \mu \mathrm{L}$ dose in normal saline. The mildly hypotonic medium in the first dose results in advective transport of antibody close to the epithelium [8], which, combined with the second dose in isotonic medium, ensures antibody was well distributed throughout the luminal layer. After another $10 \mathrm{~min}$ interval, $20 \mu \mathrm{L}$ of either PS-PEG or control PS $(0.025 \% \mathrm{w} / \mathrm{v}) \mathrm{was}$ administered in a slightly hypotonic medium ( $0.75 \mathrm{x}$ normal saline). Assuming the native volume of mCVM is approximately $20 \mu \mathrm{L}$, and that $\sim 50 \%$ of applied antibody is lost due to drip out or absorption across the vaginal epithelium due to advective flow, the final antibody concentration after two $10 \mu \mathrm{L}$ doses of $150 \mu \mathrm{g} / \mathrm{mL}$ would be $\sim 50 \mu \mathrm{g} / \mathrm{mL}$.

Mice were sacrificed after $10 \mathrm{~min}$, and the entire vagina was then gently removed and frozen in TissueTek $^{\circledR}$ O.C.T. ${ }^{\text {TM }}$ Compound (Sakura Finetek U.S.A., Inc., Torrance, CA). Transverse sections were obtained at various points along the length of the tissue (between the introitus and the cervix) with a Microm HM $500 \mathrm{M}$ Cryostat (Microm International, Walldorf, Germany). The thickness of the sections was set to 6 $\mu \mathrm{m}$ to achieve single-cell layer thickness. The sections were then stained with ProLong Gold antifade reagent (Invitrogen, Grand Island, NY) with 4',6-diamidino-2-phenylindole (DAPI) to visualize cell nuclei and retain particle fluorescence. Fluorescence images of the sections were obtained with an inverted fluorescence microscope at 10X magnification. We quantified the distribution of particles in tissue sections using Image J software (National Institutes of Health) to extract pixel intensities in the red (PSPEG) or green (PS) channel, and compared total fluorescence between the vaginal lumen and within 20 $\mu \mathrm{m}$ of the epithelial surface. The same procedures were performed for $n=3-4$ mice per condition, and particle distribution was averaged over multiple sections per mouse. Mice were anesthetized for the duration of experiments. All experimental protocols were approved by the Johns Hopkins Institutional Animal Care and Use Committee.

\subsection{Statistical analysis}

Data averages are presented as means with standard error of the mean (SEM) indicated. Statistical significance was determined by a one-tailed, Student's $t$-test $(\alpha=0.05)$.

\section{Results}

Due to both passive transudation and active transport by the MHC class I-related neonatal Fc receptor [38], IgG, rather than IgA, is the predominant immunoglobulin in human CVM secretions ( $>10-$ fold more IgG than IgA) [39]. However, the routes of PEG exposure that may lead to gradual induction of anti-PEG IgG in humans are not yet known and would be difficult to produce in mice. Therefore, we first tested whether exogenous murine anti-PEG IgG, added to ex vivo mouse CVM (mCVM) at concentrations typical of pathogen-specific antibodies in humans [25], could alter the mobility of polystyrene (PS) nanoparticles. We prepared PEGylated PS nanoparticles (PS-PEG; diameter $100 \mathrm{~nm}$ ) with PEG grafting at densities well into the dense brush regime $\left(2.3 \mathrm{PEG} / \mathrm{nm}^{2}\right)$ [28], which was corroborated by a near neutral surface charge compared to uncoated PS nanoparticles (Table 1). Due to the curvature of the nanoparticles, PEG chains likely assume a more diffuse conformation farther away from the grafted surface; this in turn suggests the polymer backbone may become increasingly exposed for antibody binding. Both anti-PEG IgG and IgM (previously shown to bind the PEG backbone) were able to bind specifically to PEG and PS-PEG nanoparticles, while control IgG and IgM did not bind (Fig. 1).

We have previously shown that PS-PEG nanoparticles undergo rapid diffusion in mCVM, while uncoated PS nanoparticles are extensively trapped and aggregated in the mucus gel due to adhesive interactions with mucins [11]. PS and PS-PEG nanoparticles used in this study exhibited similar behavior 
as previously reported (Fig. 2), which confirms the barrier properties of mCVM against mucoadhesive particles. Similar to mCVM without exogenously added IgG, in $\mathrm{mCVM}$ treated with control mouse IgG, PS-PEG exhibited largely unhindered Brownian motion, with particles capable of diffusing many microns on the order of seconds (Fig. 3A, Movie S1). However, in mCVM first treated with anti-PEG IgG to a final concentration of $10 \mu \mathrm{g} / \mathrm{mL}$ prior to the addition of nanoparticles, a large fraction of PS-PEG became immobilized (Movie S2), similar to uncoated (mucoadhesive) PS particles (Fig. 2, Movie S3). Since control IgG did not trap PEGylated nanoparticles, the observed trapping is unlikely to be caused by nonspecific binding of IgG to PEG or alterations of the mCVM microstructure due to addition of IgG antibodies. Instead, these results imply that anti-PEG antibodies can specifically bind to and trap PEGylated nanoparticles in mucus. To further validate the specificity of anti-PEG IgG binding to PEG coatings, we tested whether saturation with free PEG would prevent immobilization of the beads in mCVM. Indeed, when we added free PEG in excess of the anti-PEG antibody concentration, PS-PEG became freely mobile (Fig. S1, Movie S4).

We quantified the speeds of PS-PEG in mucus treated with different antibodies using multiple particle tracking, a technique that allows quantitative measurements of hundreds of individual particles. PS-PEG particles in control mCVM were only slowed $\sim 5$-fold compared to their theoretical speeds in water (Table 1), in good agreement with previous reports $[7,40]$. The addition of anti-PEG IgG reduced the geometrically averaged ensemble mean squared displacement ( $\langle\mathrm{MSD}\rangle)$ by $\sim 30$-fold compared to control IgG (Fig. 3B; at a time scale of $0.2667 \mathrm{~s}$ ). The impediment to free Brownian diffusion caused by anti-PEG IgG was also reflected by the slope $\alpha$ from the log-log $<M S D>v s$. time scale plots ( $\alpha=1$ for pure unobstructed Brownian diffusion, e.g., particles in water, and $\alpha$ becomes smaller and approaches zero as obstruction to Brownian diffusion increases): the average $\alpha$ value was 0.86 for PS-PEG in control CVM, but 0.52 for PS-PEG in mCVM treated with anti-PEG IgG ( $p<0.05$ vs. control). Importantly, the mobile PS-PEG fraction (see Methods) was reduced from $96 \%$ in control mCVM to only $34 \%$ in anti-PEG treated mCVM (Fig. $3 C$ ). These results demonstrate the ability for anti-PEG IgG to alter the mobility of PEGylated nanoparticles in mucus secretions.

We next sought to test whether an agglutinating antibody may trap PEGylated particles even more extensively. Secretory $\lg A(\operatorname{sg} A)$ is another common immunoglobulin found in mucus. The polymeric nature of slgA, as well as IgM, has long been suggested to facilitate "immune exclusion", the agglutination of microorganisms by polymeric immunoglobulins into clusters too large to diffuse through mucus [41]. The relevance of sIgA- and IgM-mediated immune exclusion in the female reproductive tract is vividly illustrated by agglutination of otherwise vigorously motile sperm, which make little to no forward progress after agglutination and cannot 'swim' through mucus [42]. Unfortunately, anti-PEG slgA is not commercially available and cannot be readily generated in the lab. Therefore, we evaluated the effects of anti-PEG IgM on the diffusion behavior of PEGylated nanoparticles in mucus. Addition of anti-PEG IgM immobilized an even greater fraction of PS-PEG than did anti-PEG IgG and at a lower antibody concentration ( $5 \mu \mathrm{g} / \mathrm{mL}$ vs. $10 \mu \mathrm{g} / \mathrm{mL}$; Fig. 4A and 4D, Movie S5). In contrast, addition of control IgM did not alter the diffusion of PS-PEG (Movie S6), while saturation of mCVM with free PEG prevented immobilization of PS-PEG by anti-PEG IgM (Fig. S2, Movie S7), again validating the specificity of nanoparticle trapping by anti-PEG antibodies binding to PS-PEG. The <MSD> of PS-PEG in mCVM with anti-PEG IgM was 150-fold lower than for the same particles in mCVM treated with control IgM (Fig. 4B), and reflects a drop in the mobile fraction from $97 \%$ to $7 \%$ (Fig. 4C). In comparison, mucoadhesive PS particles exhibit a mobile fraction of $3.5 \%$ on average in $\mathrm{MCVM}$ (Fig. 2). Anti-PEG IgM also reduced the average $\alpha$ value of PS-PEG from 0.79 to 0.45 ( $p<0.05$ vs. control), underscoring the extent of antibodymediated immobilization. Interestingly, we did not observe any nanoparticle agglutination in $\mathrm{mCVM}$ with anti-PEG IgM; agglutination was only observed when nanoparticles were pre-mixed with anti-PEG IgM prior to addition to mucus (Fig. S3). 
Lastly, we wanted to evaluate whether the observed changes to the diffusion of PEGylated nanoparticles in physiological mucus specimens ex vivo would also alter nanoparticle distribution at mucosal surfaces in vivo. We therefore topically administered anti-PEG or control IgM to the mouse vagina, followed by addition of PS-PEG in hypotonic medium. We observed that a substantial fraction of PS-PEG was drawn advectively through luminal mucus and accumulated immediately adjacent to the vaginal epithelium in mice dosed with control IgM, with a significant fraction penetrating deep into the rugae (Fig. 5A). In contrast, PS-PEG nanoparticles were largely trapped in luminal mucus in mice that received anti-PEG IgM; far fewer particles reached the vaginal epithelium or penetrated into the rugae (Fig. 5B), similar to mucoadhesive carboxylated latex beads (Fig. 5C). We further quantified particle distribution in the vaginal lumen vs. coating the epithelium (within $20 \mu \mathrm{m}$ of the epithelial surface). The ratio of particles in the lumen vs. epithelium was approximately 1 for PS-PEG with control IgM, indicating on average $50 \%$ of particles were within $20 \mu \mathrm{m}$ of the epithelium (Fig. 5D). In contrast, for both PS-PEG with anti-PEG IgM and uncoated latex beads, nearly 15-fold more particles were in the lumen than in proximity to the epithelium. These results are consistent with previous observations that nanoparticles that interact with and bind to the mucin mesh fibers overlaying the epithelium are unable to penetrate the mucus layer and reach the epithelium [11].

\section{Discussion}

The emergence of anti-PEG antibodies represents a potential Achilles' heel to the increasingly common use of PEG in nanomedicine. Rodent as well as large animal studies have clearly and unequivocally illustrated that anti-PEG immunity directly abrogates the extended circulation times that PEGylation generally affords to therapeutics $[17,43-46]$. Anti-PEG immunity may also result in serious complications beyond poor pharmacokinetics. Indeed, anti-PEG response as a result of repeated weekly injections of PEGylated liposomes containing synthetic oligodeoxynucleotides led to significant morbidity and mortality in mice [20]. In human clinical studies of PEG-uricase for the treatment of gout, the presence of anti-PEG antibodies was associated with early elimination and poor outcomes [47, 48]. While these studies all highlight the systemic effects associated with anti-PEG response, potential mucosal anti-PEG response has not been evaluated to date. Our finding that anti-PEG IgG or IgM can directly and specifically impede the mobility, and consequently alter the biodistribution, of PEGylated nanoparticles at mucosal surfaces adds yet another potential pitfall of anti-PEG immunity in vivo (Fig. 6).

More antibodies are secreted into mucus than into blood or lymph $[42,49,50]$; thus, a major part of the physiological immune response is likely intended to occur in the mucus secretions coating exposed surfaces of the respiratory, gastrointestinal and urogenital tracts. However, the notion that secreted antibodies can work together with mucus to impede the mobility of pathogens and foreign particulates and reduce the flux arriving at the epithelium remains largely unrecognized. This is in part a consequence of the exceedingly weak affinity between antibodies and mucus, which would suggest that individual antibodies are incapable of crosslinking a pathogen or particle to mucus. It was not until very recently that virus-binding IgG antibodies were shown by both experiments [25] and by computational modeling [27] to be capable of immobilizing viruses in mucus via multiple bonds between mucins and $\mathrm{N}$ glycans on IgG-Fc, which in turn reduces the flux of viruses arriving at the epithelium in vivo. Our observed correlation between limited PEG-coated nanoparticle diffusion due to anti-PEG antibodies in mucus and their exclusion from reaching the vaginal epithelium is consistent with the poor mucus penetration and distribution observed previously with conventional latex nanoparticles in the mouse vagina [11]. The immobilization of PEGylated nanoparticles by anti-PEG antibodies in MCVM offers further evidence that trapping by antibody-mucin crosslinking may be a universal mechanism of mucosal immunity across different animal species.

We found that anti-PEG IgM was substantially more potent in trapping individual PEGylated nanoparticles in mucus than anti-PEG IgG. This may be due to a number of reasons. First, anti-PEG IgM 
has a higher binding avidity to PEG than anti-PEG IgG (see Methods), which likely translates to greater antibody accumulation on the surface of PEGylated nanoparticles. Second, IgM likely possesses greater avidity to mucins than IgG. This can be inferred from the diffusivity of individual IgM molecules in mucus, which is slowed nearly $50 \%$ compared to in buffer, whereas individual IgG molecules are slowed only $5-20 \%$ in mucus compared to in buffer [26]. Because both IgG and IgM are far too small relative to the mucin mesh spacing [7] to be slowed substantially by steric hindrance, IgM must be slowed to a greater extent than IgG due to more and/or stronger adhesive interactions with mucus constituents. The greater mucin-binding avidity of IgM implies fewer particle-bound IgM would be needed to immobilize a PEGylated nanoparticle compared to IgG; indeed, greater trapping of PS-PEG was achieved with IgM than IgG even at a lower concentration.

A classical mechanism of immune defense is "immune exclusion", which describes the agglutination of microorganisms in the gut by secreted polyvalent IgA or IgM into clusters too large to diffuse through mucus [51]. Although mucus is highly viscoelastic at the macroscale [1, 52], PEGylated nanoparticles can readily diffuse through mucus at rates comparable to their rates in water [4, 9], and thus are not precluded from colliding with each other and becoming agglutinated. Interestingly, we observed negligible levels of agglutination in all of our microscopy studies. This is likely because even a single particle-bound IgM can substantially impede the Brownian motion of the particle (assuming the binding of IgM to PEG does not alter IgM affinity to mucins, a single IgM bound to the nanoparticle may slow it down by $\sim 50 \%$ ), and lower particle mobility directly reduces the frequency of colliding with other particles, a necessary first-step to agglutination. Thus, IgM-induced agglutination is unlikely to be prevalent when the kinetics of IgM accumulating onto the nanoparticle surface is substantially faster than the rate of collision between nanoparticles. This directly challenges the general dogma that antibody-mediated agglutination - often supported by micrographs showing the formation of large immune complexes upon incubation of antibodies and pathogens in buffer in the absence of mucus - is a dominant mechanism of mucosal immunity by polymeric immunoglobulins.

In addition to a lack of standardized quantitative assays to characterize anti-PEG antibodies, a major contention to reports of anti-PEG phenomenon in the literature is that the observations may not require antibodies that specifically bind PEG polymer [22]. Indeed, Schellekens et al. suggested that the accelerated blood clearance observed in many studies could perhaps be attributed to complement activation and/or non-specific accumulation of IgM and IgG onto the surface of PEGylated drugs or drug carriers. Although it is impossible to draw direct comparisons between studies performed in blood vs. mucus, we showed here that the mobility of PEG-coated nanoparticles was specifically retarded by antiPEG IgG and IgM, because (i) addition of control IgG and IgM antibodies did not comparably alter bead motions, (ii) addition of free PEG competitively inhibits trapping of beads by anti-PEG antibodies, and (iii) the total dose of anti-PEG IgG or IgM in mucus represents only a very small fraction of the total IgG and IgM present [25]. Although it is likely that not all reports of accelerated blood clearance of PEGylated agents are caused solely by anti-PEG antibodies, our studies underscore the ability of antiPEG IgG and IgM to specifically bind PEGylated particles in physiologically relevant environments (i.e. mucus), and in turn alter the biological fate of the PEGylated particles.

The concentration of approximately $50 \mu \mathrm{g} / \mathrm{mL}$ anti-PEG antibody used in our in vivo studies (see Methods) is likely in the range of concentrations that can be achieved under physiological conditions. Kiwada and coworkers recently showed that $\sim 50 \mu \mathrm{g} / \mathrm{mL}$ of serum anti-PEG IgM can be induced following injection of PEG-liposomes at a dose of $5 \mathrm{nmol}$ of phospholipids per mouse [53]. Much of the antibody in secretions coating the vaginal epithelium is derived from serum transudation, and thus is likely similar in composition to the systemic antibody repertoire. Indeed, despite the common belief that IgA is the main immunoglobulin found in mucus, IgG is in fact the dominant antibody isotype in vaginal secretions $[38,39]$, similar to in serum. In addition, although PEG-specific antibody levels in human vaginal 
secretions have never been measured, we have previously found Herpes-specific antibodies at up to 20$30 \mu \mathrm{g} / \mathrm{mL}$ levels in native vaginal secretions from human donors [54].

The vast majority of the literature on anti-PEG immunity focuses on the acute induction of antiPEG immune response by specific PEGylated liposomes or nanoparticle therapeutics. The possibility of pre-existing anti-PEG response is rarely addressed. Because most of the population has yet to receive PEGylated therapeutics, the presence of PEG-specific antibodies in bodily secretions such as mucus most likely results from induction by alternative routes of exposure. Since PEG and other PEG-containing polymers are often found in soaps and other detergents, a hypothetical route of exposure could be via open cuts and wounds cleaned with PEG-containing soaps, or bleeding gums at the oral mucosa exposed to PEG-containing toothpaste. The combination of PEG and surfactants that are toxic to cells may provide a danger signal that inadvertently induces immune cells to generate antibodies against PEG. Alternatively, PEG is also frequently found in numerous foods as well as oral and topical products. Repeated exposure to PEG at the oral, gastrointestinal and vaginal mucosa, including exposure accompanied by surfactants, could potentially induce systemic and/or mucosal anti-PEG immunity over time, with significant implications for the delivery of PEGylated therapeutics via relevant routes of administration.

\section{Conclusion}

PEG is widely used in nanomedicine as a stealth coating polymer to improve circulation time and therapeutic efficacy. However, a growing body of evidence generated over the past two decades shows that systemic exposure to PEG can induce anti-PEG antibodies that not only significantly alter the systemic circulation kinetics of PEG-modified nanotherapeutics, but may also directly result in adverse outcomes. Our finding that anti-PEG antibodies can also trap PEG-coated nanoparticles in mucus secretions ex vivo and alter particle biodistribution at mucosal surfaces in vivo further suggests that mucosal anti-PEG immunity may be an under-recognized challenge. Mucosal exposure to PEG and the prevalence of anti-PEG antibodies in human mucus is not yet well understood. In light of the large arsenal of PEGylated therapeutics that are FDA-approved or in clinical development for both systemic and mucosal applications, we believe further investigations into the origin and characteristics of antiPEG immunity are of critical importance to support ongoing efforts in translational nanomedicine development.

\section{Acknowledgement}

Financial support was provided by the PhRMA Foundation Predoctoral Fellowship (Q.Y.), National Science Foundation CAREER Award (DMR-1151477, S.K.L.), The David and Lucile Packard Foundation (2013-39274, S.K.L.), National Institutes of Health grants R21EB017938 (S.K.L.), U19AI096398 (S.K.L. and R.C.), 1F32Al102535 (YYW), and Diversity Supplement Al096398 S1 (KLN), and startup funds from the Eshelman School of Pharmacy and Lineberger Comprehensive Cancer Center (S.K.L.). The funders had no role in study design, data collection and analysis, decision to publish, or preparation of the manuscript. We thank Durai B. Subramani for technical assistance.

\section{Disclosures}

Mucommune LLC seeks to harness antibody-mucin interactions to improve protection or treatment of infections at mucosal surfaces, and has licensed intellectual property from the University of North Carolina - Chapel Hill (UNC-CH). SKL is a founder of Mucommune. SKL and YYW own company stock; SKL's relationship with Mucommune is subject to certain restrictions under University policy. The terms of this arrangement are being managed by $\mathrm{UNC}-\mathrm{CH}$ in accordance with its conflict of interest policies.

\section{References}


[1] Lai SK, Wang YY, Wirtz D, Hanes J. Micro- and macrorheology of mucus. Advanced drug delivery reviews 2009;61:86-100.

[2] Lai SK, Hida K, Shukair S, Wang YY, Figueiredo A, Cone R, Hope TJ, Hanes J. Human immunodeficiency virus type 1 is trapped by acidic but not by neutralized human cervicovaginal mucus. Journal of virology 2009;83:11196-200.

[3] Cu Y, Saltzman WM. Controlled surface modification with poly(ethylene)glycol enhances diffusion of PLGA nanoparticles in human cervical mucus. Molecular pharmaceutics 2009;6:173-81.

[4] Lai SK, O'Hanlon DE, Harrold S, Man ST, Wang YY, Cone R, Hanes J. Rapid transport of large polymeric nanoparticles in fresh undiluted human mucus. Proc Natl Acad Sci USA 2007;104:1482-7.

[5] Abdulkarim M, Agullo N, Cattoz B, Griffiths P, Bernkop-Schnurch A, Borros SG, Gumbleton M. Nanoparticle diffusion within intestinal mucus: Three-dimensional response analysis dissecting the impact of particle surface charge, size and heterogeneity across polyelectrolyte, pegylated and viral particles. European journal of pharmaceutics and biopharmaceutics : official journal of Arbeitsgemeinschaft fur Pharmazeutische Verfahrenstechnik eV 2015.

[6] Macierzanka A, Rigby NM, Corfield AP, Wellner N, Bottger F, Mills ENC, Mackie AR. Adsorption of bile salts to particles allows penetration of intestinal mucus. Soft Matter 2011;7:8077-84.

[7] Lai SK, Wang YY, Hida K, Cone R, Hanes J. Nanoparticles reveal that human cervicovaginal mucus is riddled with pores larger than viruses. Proc Natl Acad Sci U S A 2010;107:598-603.

[8] Ensign LM, Hoen TE, Maisel K, Cone RA, Hanes JS. Enhanced vaginal drug delivery through the use of hypotonic formulations that induce fluid uptake. Biomaterials 2013;34:6922-9.

[9] Wang YY, Lai SK, Suk JS, Pace A, Cone R, Hanes J. Addressing the PEG mucoadhesivity paradox to engineer nanoparticles that "slip" through the human mucus barrier. Angewandte Chemie (International ed 2008;47:9726-9.

[10] Yang M, Yu T, Wang YY, Lai SK, Zeng Q, Miao B, Tang BC, Simons BW, Ensign LM, Liu G, Chan KW, Juang CY, Mert O, Wood J, Fu J, McMahon MT, Wu TC, Hung CF, Hanes J. Vaginal delivery of paclitaxel via nanoparticles with non-mucoadhesive surfaces suppresses cervical tumor growth. Advanced healthcare materials 2014;3:1044-52.

[11] Ensign LM, Tang BC, Wang YY, Tse TA, Hoen T, Cone R, Hanes J. Mucus-penetrating nanoparticles for vaginal drug delivery protect against herpes simplex virus. Science translational medicine 2012;4:138ra79.

[12] Suk JS, Kim AJ, Trehan K, Schneider CS, Cebotaru L, Woodward OM, Boylan NJ, Boyle MP, Lai SK, Guggino WB, Hanes J. Lung gene therapy with highly compacted DNA nanoparticles that overcome the mucus barrier. Journal of controlled release : official journal of the Controlled Release Society 2014;178:8-17.

[13] Allen TM, Hansen C, Martin F, Redemann C, Yau-Young A. Liposomes containing synthetic lipid derivatives of poly(ethylene glycol) show prolonged circulation half-lives in vivo. Biochim Biophys Acta 1991;1066:29-36.

[14] Blume G, Cevc G. Liposomes for the sustained drug release in vivo. Biochim Biophys Acta 1990;1029:91-7.

[15] Klibanov AL, Maruyama K, Torchilin VP, Huang L. Amphipathic polyethyleneglycols effectively prolong the circulation time of liposomes. FEBS Lett 1990;268:235-7.

[16] Senior J, Delgado C, Fisher D, Tilcock C, Gregoriadis G. Influence of surface hydrophilicity of liposomes on their interaction with plasma protein and clearance from the circulation: studies with poly(ethylene glycol)-coated vesicles. Biochim Biophys Acta 1991;1062:77-82.

[17] Dams ET, Laverman P, Oyen WJ, Storm G, Scherphof GL, van Der Meer JW, Corstens FH, Boerman OC. Accelerated blood clearance and altered biodistribution of repeated injections of sterically stabilized liposomes. The Journal of pharmacology and experimental therapeutics 2000;292:1071-9. 
[18] Ishida T, Masuda K, Ichikawa T, Ichihara M, Irimura K, Kiwada H. Accelerated clearance of a second injection of PEGylated liposomes in mice. International journal of pharmaceutics 2003;255:167-74.

[19] Judge A, McClintock K, Phelps JR, Maclachlan I. Hypersensitivity and loss of disease site targeting caused by antibody responses to PEGylated liposomes. Molecular therapy : the journal of the American Society of Gene Therapy 2006;13:328-37.

[20] Semple SC, Harasym TO, Clow KA, Ansell SM, Klimuk SK, Hope MJ. Immunogenicity and rapid blood clearance of liposomes containing polyethylene glycol-lipid conjugates and nucleic Acid. The Journal of pharmacology and experimental therapeutics 2005;312:1020-6.

[21] Wang X, Ishida T, Kiwada H. Anti-PEG IgM elicited by injection of liposomes is involved in the enhanced blood clearance of a subsequent dose of PEGylated liposomes. Journal of controlled release : official journal of the Controlled Release Society 2007;119:236-44.

[22] Schellekens $\mathrm{H}$, Hennink WE, Brinks V. The immunogenicity of polyethylene glycol: facts and fiction. Pharmaceutical research 2013;30:1729-34.

[23] Armstrong JK, Leger R, Wenby RB, Meiselman HJ, Garratty G, Fisher TC. Occurrence of an antibody to poly(ethylene glycol) in normal donors. Blood 2003;102:556.

[24] Yang Q, Lai SK. Anti-PEG immunity: emergence, characteristics, and unaddressed questions. Wiley interdisciplinary reviews Nanomedicine and nanobiotechnology 2015;7:655-77.

[25] Wang YY, Kannan A, Nunn KL, Murphy MA, Subramani DB, Moench T, Cone R, Lai SK. IgG in cervicovaginal mucus traps HSV and prevents vaginal herpes infections. Mucosal immunology 2014;7:1036-44.

[26] Olmsted SS, Padgett JL, Yudin Al, Whaley KJ, Moench TR, Cone RA. Diffusion of macromolecules and virus-like particles in human cervical mucus. Biophys J 2001;81:1930-7.

[27] Chen A, McKinley SA, Wang S, Shi F, Mucha PJ, Forest MG, Lai SK. Transient antibody-mucin interactions produce a dynamic molecular shield against viral invasion. Biophys J 2014;106:2028-36.

[28] Yang Q, Jones SW, Parker CL, Zamboni WC, Bear JE, Lai SK. Evading immune cell uptake and clearance requires PEG grafting at densities substantially exceeding the minimum for brush conformation. Molecular pharmaceutics 2014;11:1250-8.

[29] Academia Sinica. http://www.ibms.sinica.edu.tw/ sroff/anti-PEG/anti-PEG_antibody_order.html. Accessed February 22, 2016.

[30] Su YC, Chen BM, Chuang KH, Cheng TL, Roffler SR. Sensitive quantification of PEGylated compounds by second-generation anti-poly(ethylene glycol) monoclonal antibodies. Bioconjugate chemistry 2010;21:1264-70.

[31] Su YC, Al-Qaisi TS, Tung HY, Cheng TL, Chuang KH, Chen BM, Roffler SR. Mimicking the germinal center reaction in hybridoma cells to isolate temperature-selective anti-PEG antibodies. mAbs 2014;6:1069-83.

[32] Ehrlich GK, Michel H, Chokshi HP, Malick AW. Affinity purification and characterization of an antiPEG IgM. Journal of molecular recognition : JMR 2009;22:99-103.

[33] Apgar J, Tseng Y, Fedorov E, Herwig MB, Almo SC, Wirtz D. Multiple-particle tracking measurements of heterogeneities in solutions of actin filaments and actin bundles. Biophys $J$ 2000;79:1095-106.

[34] Wang YY, Nunn KL, Harit D, McKinley SA, Lai SK. Minimizing biases associated with tracking analysis of submicron particles in heterogeneous biological fluids. Journal of controlled release : official journal of the Controlled Release Society 2015;220:37-43.

[35] Dawson M, Wirtz D, Hanes J. Enhanced viscoelasticity of human cystic fibrotic sputum correlates with increasing microheterogeneity in particle transport. J Biol Chem 2003;278:50393-401.

[36] Suh J, Dawson M, Hanes J. Real-time multiple-particle tracking: applications to drug and gene delivery. Advanced drug delivery reviews 2005;57:63-78. 
[37] Lai SK, O'Hanlon DE, Harrold S, Man ST, Wang YY, Cone R, Hanes J. Rapid transport of large polymeric nanoparticles in fresh undiluted human mucus. Proceedings of the National Academy of Sciences of the United States of America 2007;104:1482-7.

[38] Li Z, Palaniyandi S, Zeng R, Tuo W, Roopenian DC, Zhu X. Transfer of IgG in the female genital tract by $\mathrm{MHC}$ class I-related neonatal Fc receptor $(\mathrm{FcRn})$ confers protective immunity to vaginal infection. Proc Natl Acad Sci U S A 2011;108:4388-93.

[39] Usala SJ, Usala FO, Haciski R, Holt JA, Schumacher GF. IgG and IgA content of vaginal fluid during the menstrual cycle. The Journal of reproductive medicine 1989;34:292-4.

[40] Ensign LM, Henning A, Schneider CS, Maisel K, Wang YY, Porosoff MD, Cone R, Hanes J. Ex vivo characterization of particle transport in mucus secretions coating freshly excised mucosal tissues. Molecular pharmaceutics 2013;10:2176-82.

[41] Roche AM, Richard AL, Rahkola JT, Janoff EN, Weiser JN. Antibody blocks acquisition of bacterial colonization through agglutination. Mucosal Immunol 2014.

[42] Cone RA. Mucus. In: Ogra PL, Mestecky J, Lamm ME, Strober W, Bienenstock J, McGhee JR, editors. Handbook of Mucosal Immunology. 3 ed. San Diego: Academic Press; 1999. p. 43-64.

[43] Ishida T, Ichihara M, Wang X, Yamamoto K, Kimura J, Majima E, Kiwada H. Injection of PEGylated liposomes in rats elicits PEG-specific IgM, which is responsible for rapid elimination of a second dose of PEGylated liposomes. Journal of controlled release : official journal of the Controlled Release Society 2006;112:15-25.

[44] Mima Y, Hashimoto Y, Shimizu T, Kiwada H, Ishida T. Anti-PEG IgM Is a Major Contributor to the Accelerated Blood Clearance of Polyethylene Glycol-Conjugated Protein. Molecular pharmaceutics 2015;12:2429-35.

[45] Suzuki T, Ichihara M, Hyodo K, Yamamoto E, Ishida T, Kiwada H, Ishihara H, Kikuchi H. Accelerated blood clearance of PEGylated liposomes containing doxorubicin upon repeated administration to dogs. International journal of pharmaceutics 2012;436:636-43.

[46] Suzuki T, Ichihara M, Hyodo K, Yamamoto E, Ishida T, Kiwada H, Kikuchi H, Ishihara H. Influence of dose and animal species on accelerated blood clearance of PEGylated liposomal doxorubicin. International journal of pharmaceutics 2014;476:205-12.

[47] Ganson NJ, Kelly SJ, Scarlett E, Sundy JS, Hershfield MS. Control of hyperuricemia in subjects with refractory gout, and induction of antibody against poly(ethylene glycol) (PEG), in a phase I trial of subcutaneous PEGylated urate oxidase. Arthritis research \& therapy 2006;8:R12.

[48] Sundy JS, Ganson NJ, Kelly SJ, Scarlett EL, Rehrig CD, Huang W, Hershfield MS. Pharmacokinetics and pharmacodynamics of intravenous PEGylated recombinant mammalian urate oxidase in patients with refractory gout. Arthritis and rheumatism 2007;56:1021-8.

[49] Conley ME, Delacroix DL. Intravascular and mucosal immunoglobulin A: two separate but related systems of immune defense? Annals of internal medicine 1987;106:892-9.

[50] Mestecky J, Russell MW, Jackson S, Brown TA. The human IgA system: a reassessment. Clinical immunology and immunopathology 1986;40:105-14.

[51] Mestecky J, Russell MW, Elson CO. Intestinal IgA: novel views on its function in the defence of the largest mucosal surface. Gut 1999;44:2-5.

[52] Lai SK, Wang YY, Cone R, Wirtz D, Hanes J. Altering mucus rheology to "solidify" human mucus at the nanoscale. PloS one 2009;4:e4294.

[53] Hashimoto Y, Shimizu T, Abu Lila AS, Ishida T, Kiwada H. Relationship between the concentration of anti-polyethylene glycol (PEG) immunoglobulin $\mathrm{M}$ (IgM) and the intensity of the accelerated blood clearance $(A B C)$ phenomenon against PEGylated liposomes in mice. Biological \& pharmaceutical bulletin 2015;38:417-24.

[54] Wang YY, Kannan A, Nunn KL, Murphy MA, Subramani DB, Moench T, Cone R, Lai SK. IgG in cervicovaginal mucus traps HSV and prevents vaginal Herpes infections. Mucosal Immunol 2014. 
[55] Saltzman WM, Radomsky ML, Whaley KJ, Cone RA. Antibody diffusion in human cervical mucus. Biophys J 1994;66:508-15.

\section{Figure Legends}

Figure 1. Specificity of anti-PEG antibody binding to PEG. (A,B) Binding of (A) anti-PEG or control IgG and (B) anti-PEG or control IgM antibodies to PEG-coated plates at different concentrations of free $\mathrm{PEG}_{8 \mathrm{k}}$. (C-F) Dot blot assay demonstrating binding of anti-PEG antibodies to PS-PEG vs. control PS beads. PS and PS-PEG beads were blotted onto nitrocellulose membrane and incubated with (C) anti-PEG IgG, (D) control IgG, (E) anti-PEG IgM, or (F) control lgM.

Figure 2. Diffusion rates of uncoated or PEG-coated PS nanoparticles in mCVM. (A) Representative trajectories for particles exhibiting effective diffusivities within one SEM of the ensemble average at a time scale of $0.2667 \mathrm{~s}$. (B) Ensemble-averaged geometric mean square displacements ( $\langle\mathrm{MSD}\rangle)$ as a function of time scale. ${ }^{*}$ indicates a statistically significant difference $(p<0.05)$. (C) Distributions of the logarithms of individual particle effective diffusivities $\left(D_{\text {eff }}\right)$ at a time scale of $0.2667 \mathrm{~s}$. Log $D_{\text {eff }}$ values to the left of the dashed line correspond to particles with displacements of less than $\sim 100 \mathrm{~nm}$ (i.e., less than the particle diameter) within $0.2667 \mathrm{~s}$. These small motions are consistent with particles permanently stuck to the mucus gel, and most likely reflect thermal motions of the gel itself. Data represent the ensemble average of three independent experiments, with $n \geq 40$ particles per frame $(n \geq$ 100 particle traces per experiment) on average for each experiment.

Figure 3. Diffusion rates of PEG-coated nanoparticles in $\mathrm{MCVM}$ treated with different IgG antibodies. (A) Representative trajectories for particles exhibiting effective diffusivities within one SEM of the ensemble average at a time scale of $0.2667 \mathrm{~s}$. (B) Ensemble-averaged geometric mean square displacements $(<M S D>)$ as a function of time scale. ${ }^{*}$ indicates a statistically significant difference $(p<0.05)$. (C) Distributions of the logarithms of individual particle effective diffusivities $\left(D_{\text {eff }}\right)$ at a time scale of 0.2667 s. Log $D_{\text {eff }}$ values to the left of the dashed line correspond to particles with displacements of less than $\sim 100 \mathrm{~nm}$ (i.e., less than the particle diameter) within $0.2667 \mathrm{~s}$. Data represent the ensemble average of four independent experiments, with $n \geq 40$ particles per frame ( $n \geq 100$ particle traces per experiment) on average for each experiment.

Figure 4. Diffusion rates of PEG-coated nanoparticles in $\mathrm{mCVM}$ treated with different IgM antibodies. (A) Representative trajectories for particles exhibiting effective diffusivities within one SEM of the ensemble average at a time scale of $0.2667 \mathrm{~s}$. (B) Ensemble-averaged geometric mean square displacements $(\langle M S D\rangle)$ as a function of time scale. (C) Distributions of the logarithms of individual particle effective diffusivities ( $D_{\text {eff }}$ ) at a time scale of $0.2667 \mathrm{~s}$. Log $D_{\text {eff }}$ values to the left of the dashed line correspond to particles that are effectively trapped, with displacements of less than $100 \mathrm{~nm}$ (i.e., less than the particle diameter) within $0.2667 \mathrm{~s}$. (D) Ensemble averaged geometric effective diffusion coefficients at a timescale of $0.2667 \mathrm{~s}$ for mucus treated with different IgG and IgM antibodies. Distinct samples are indicated with different color circles; averages are indicated by solid lines. ${ }^{*}$ indicates a statistically significant difference $(p<0.05)$. Data represent the ensemble average of four independent experiments, with $n \geq 40$ particles per frame ( $n \geq 100$ particle traces per experiment) on average for each experiment. 
Figure 5. Representative transverse $50 \mu \mathrm{m}$ thick frozen tissue sections showing distribution of $(A, B)$ PEGcoated nanoparticles in the mouse vagina treated with (A) control or (B) anti-PEG antibody, or of (C) uncoated PS nanoparticles in the mouse vagina. Red corresponds to PEG-coated nanoparticles, green corresponds to uncoated PS nanoparticles, and blue corresponds to DAPI-stained cell nuclei. Scale bars indicate a length of $200 \mu \mathrm{m}$. (D) Image-based quantification of particle distribution in the vaginal lumen vs. in close proximity to the epithelium (within $20 \mu \mathrm{m}$ of the epithelial surface). * indicates a statistically significant difference $(p<0.05)$.

Figure 6. Schematic illustrating the possible effects of anti-PEG antibodies in mucus on PEG-coated nanoparticles administered to the vaginal mucosal surface consistent with the observed particle distribution in Figure 5. In the absence of specific antibodies, PEG-coated nanoparticles can diffuse quickly through the mucus layer and reach the vaginal epithelium as well as enter into the rugae (folds in the vaginal epithelium), thereby achieving more uniform coverage of the entire epithelial surface, as demonstrated previously $[8,11]$. When anti-PEG antibodies are present in mucus, PEG-coated nanoparticles are captured by anti-PEG antibodies that, despite individually weak bonds with mucins $[26,55]$ (indicated by arrows), can collectively generate sufficient avidity to trap the nanoparticle in mucus, similar to the trapping of viruses by virus-specific antibodies in mucus $[25,27]$. PEG-coated nanoparticles immobilized in mucus are localized largely within the mucus layer rather than in close proximity to the vaginal epithelium, akin to uncoated (mucoadhesive) nanoparticles [11]. 
Table 1. Characterization of PEG-modified nanoparticles, and ratios of the ensemble average diffusion coefficients in water $\left(D_{\mathrm{w}}\right)$ compared to in $\operatorname{mCVM}\left(D_{\mathrm{m}}\right)$. Size and $\zeta$-potential values for carboxyl-modified beads are provided for comparison.

\begin{tabular}{llllll}
\hline Size $(\mathrm{nm})^{*}$ & $\begin{array}{l}\text { Surface } \\
\text { Chemistry }\end{array}$ & Diameter $(\mathrm{nm})$ & $\begin{array}{l}\zeta \text {-potential } \\
(\mathrm{mV})\end{array}$ & $\begin{array}{l}\text { PEG Density } \\
\left(\mathrm{PEG} / \mathrm{nm}^{2}\right) \dagger\end{array}$ & $D_{\mathrm{w}} / D_{\mathrm{m}} \ddagger$ \\
\hline 100 & COOH & $109 \pm 4$ & $-55 \pm 5$ & $\mathrm{~N} / \mathrm{A}$ & $\mathrm{N} / \mathrm{A}$ \\
100 & PEG & $132 \pm 3$ & $-7 \pm 3$ & $2.3 \pm 0.1$ & 4.9 \\
\hline
\end{tabular}

* Provided by the manufacturer.

+ Calculated from \% $\mathrm{COOH}$ substitution measured by PDAM assay.

¥ Effective diffusivity values are calculated at a time scale of $0.2667 \mathrm{~s}$. $D_{\mathrm{w}}$ is calculated from the StokesEinstein equation. 
Figure 1

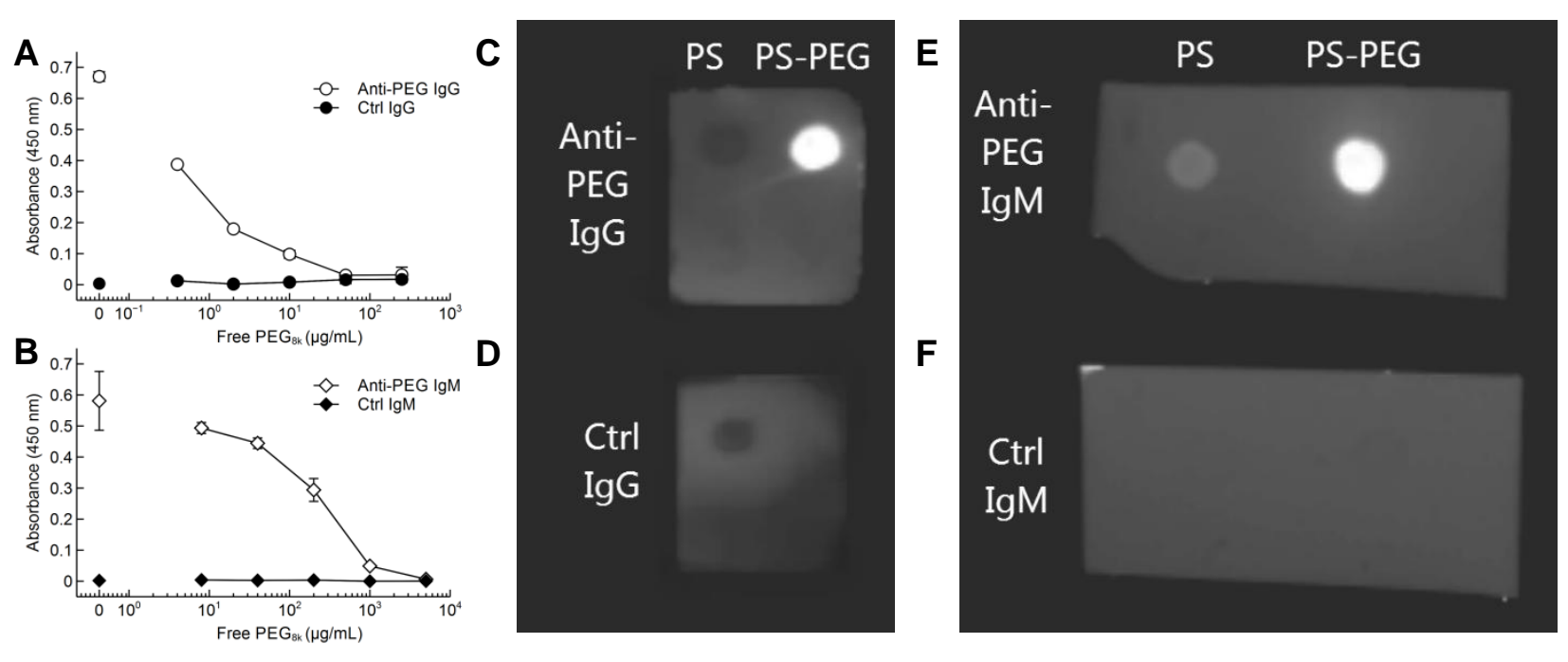


Figure 2

A

PS

PS-PEG

$\frac{\bullet}{1 \mu \mathrm{m}}$

B
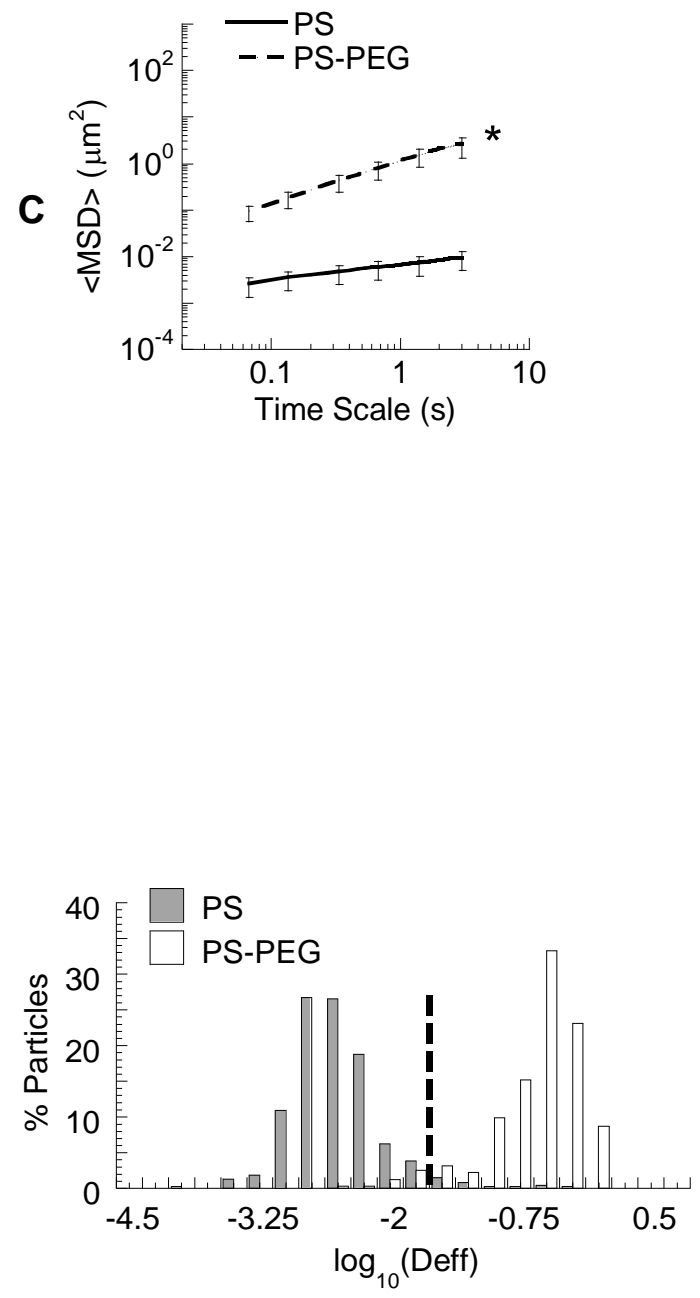

Figure 3

A Control lgG Anti-PEG IgG
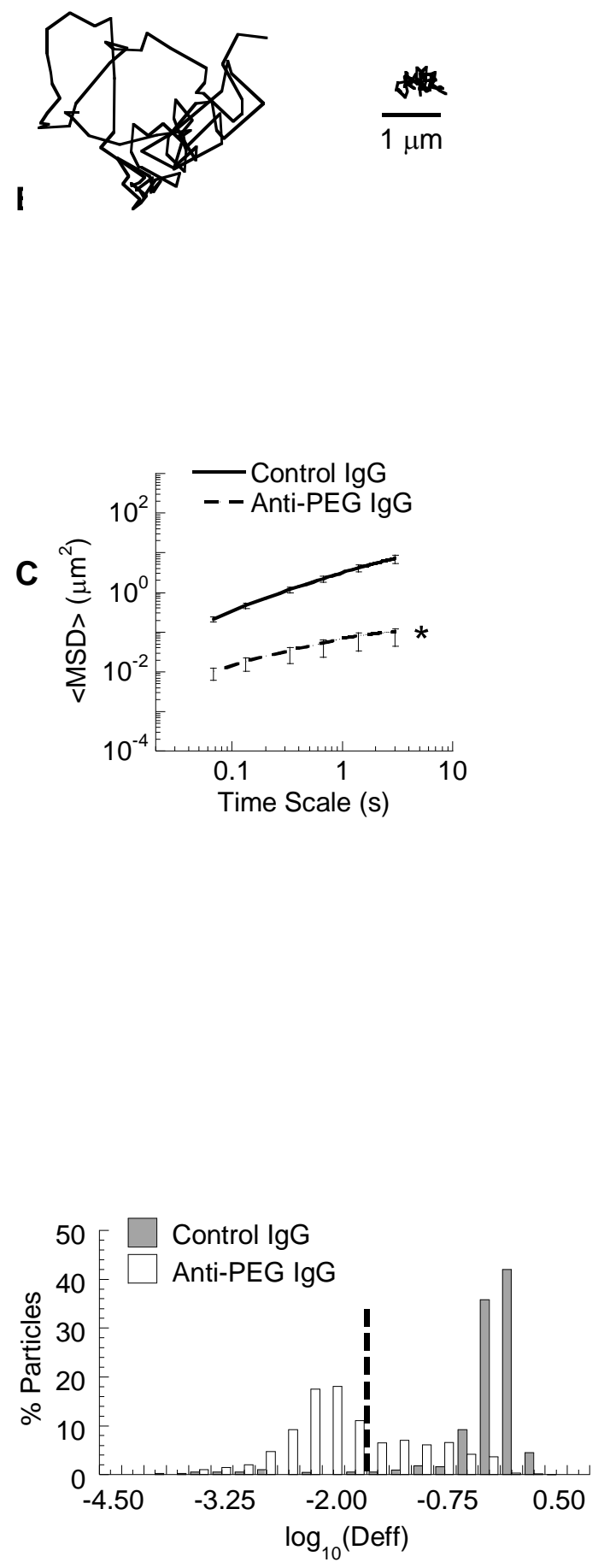
Figure 4

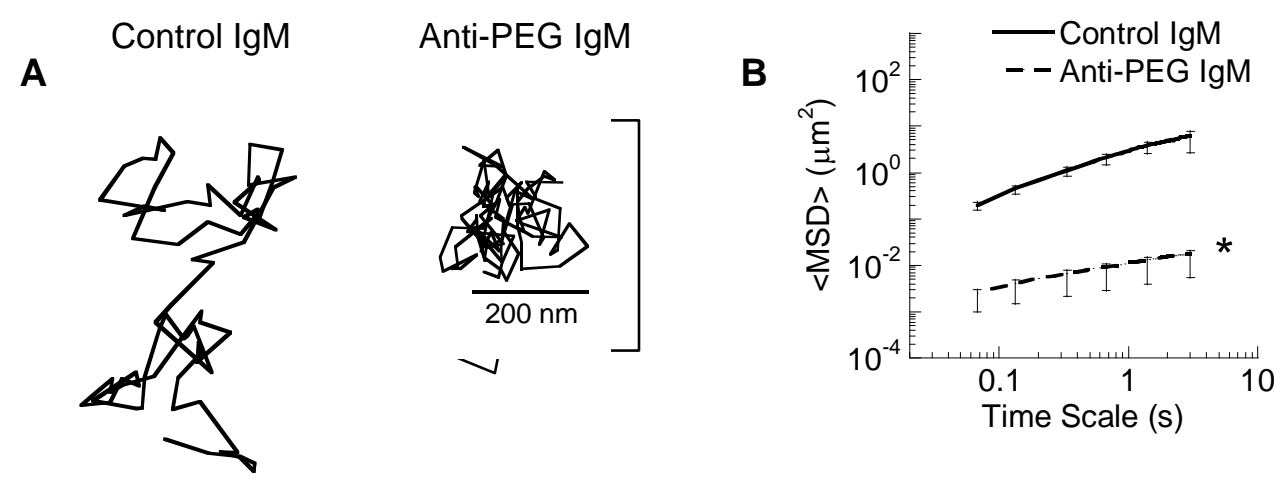

$\frac{\boldsymbol{x}}{1 \mu \mathrm{m}}$
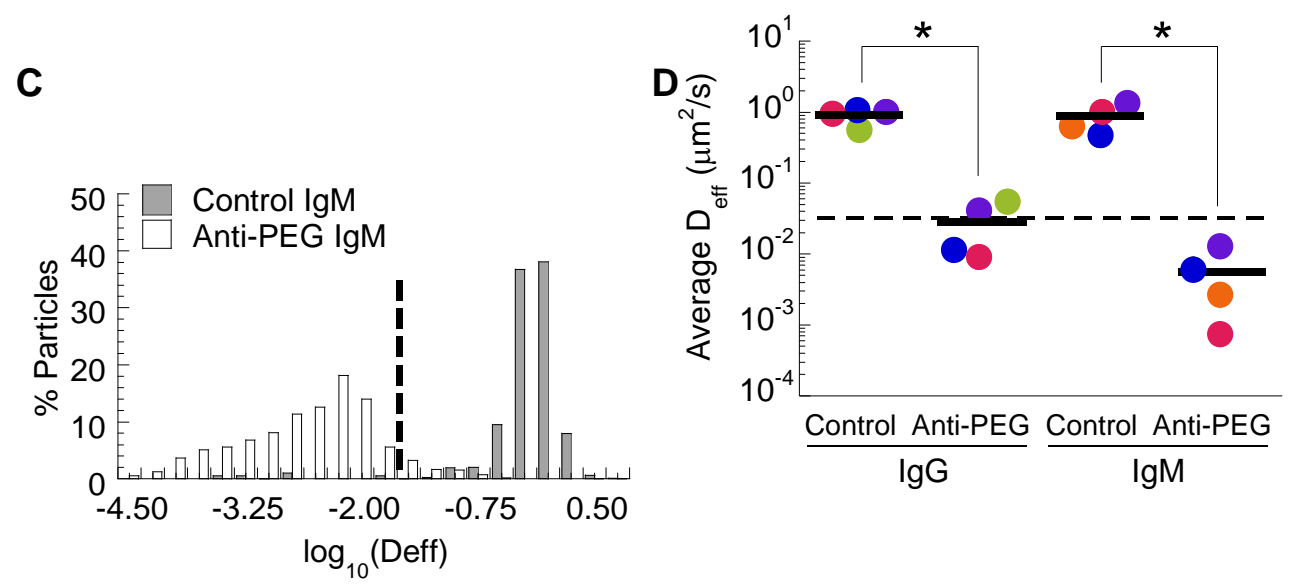
Figure 5

A PS-PEG + Control Ab

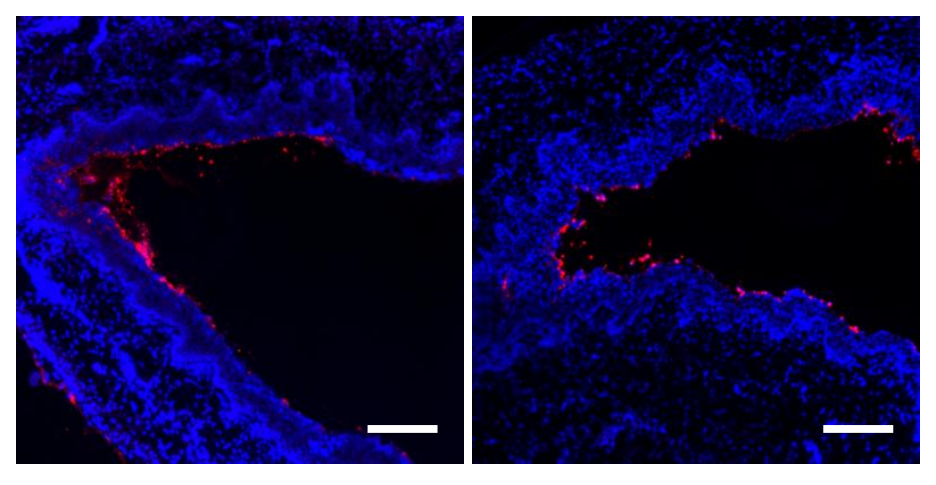

B $\quad$ PS-PEG + Anti-PEG Ab

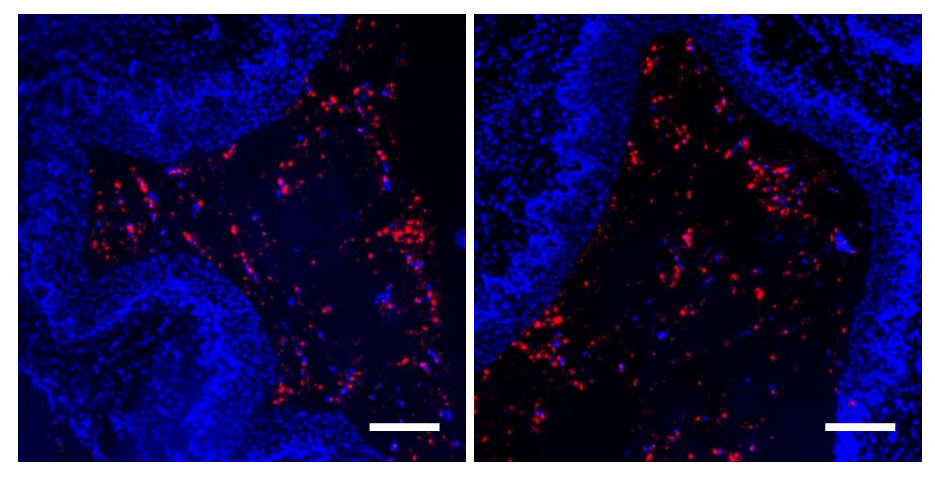

C Uncoated PS

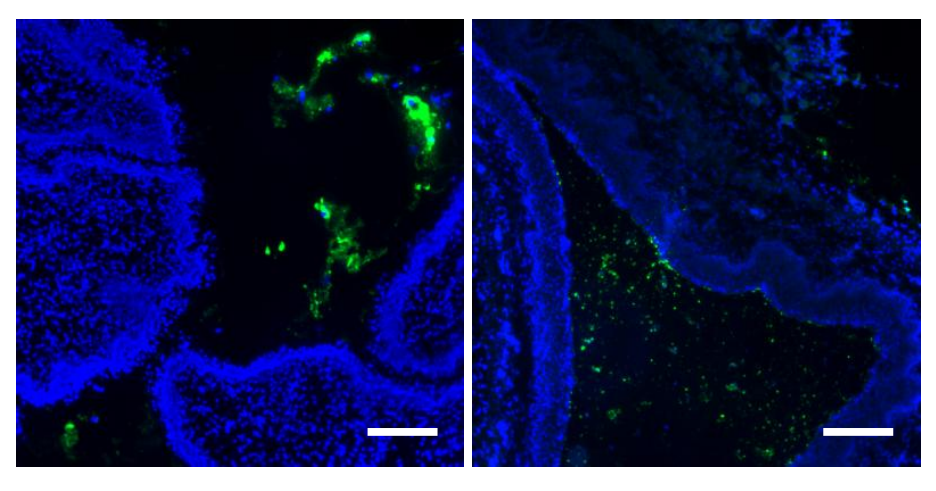

D

Page 21 of 23 


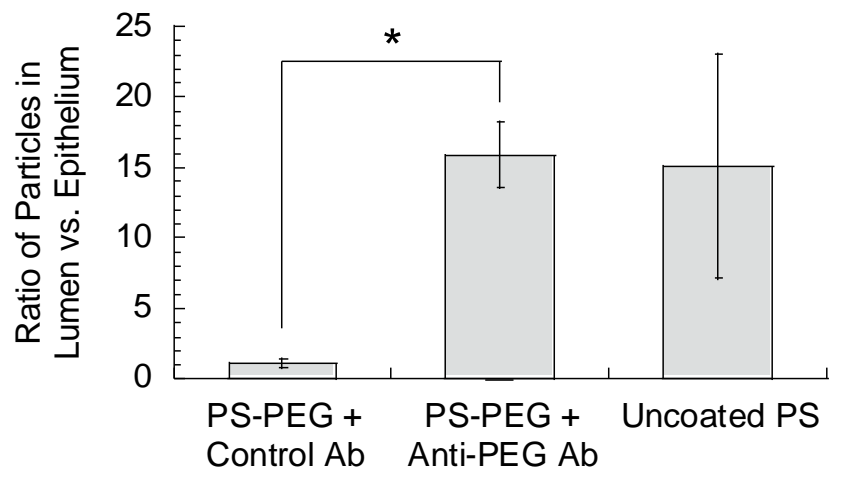

Page 22 of 23 
Figure 6

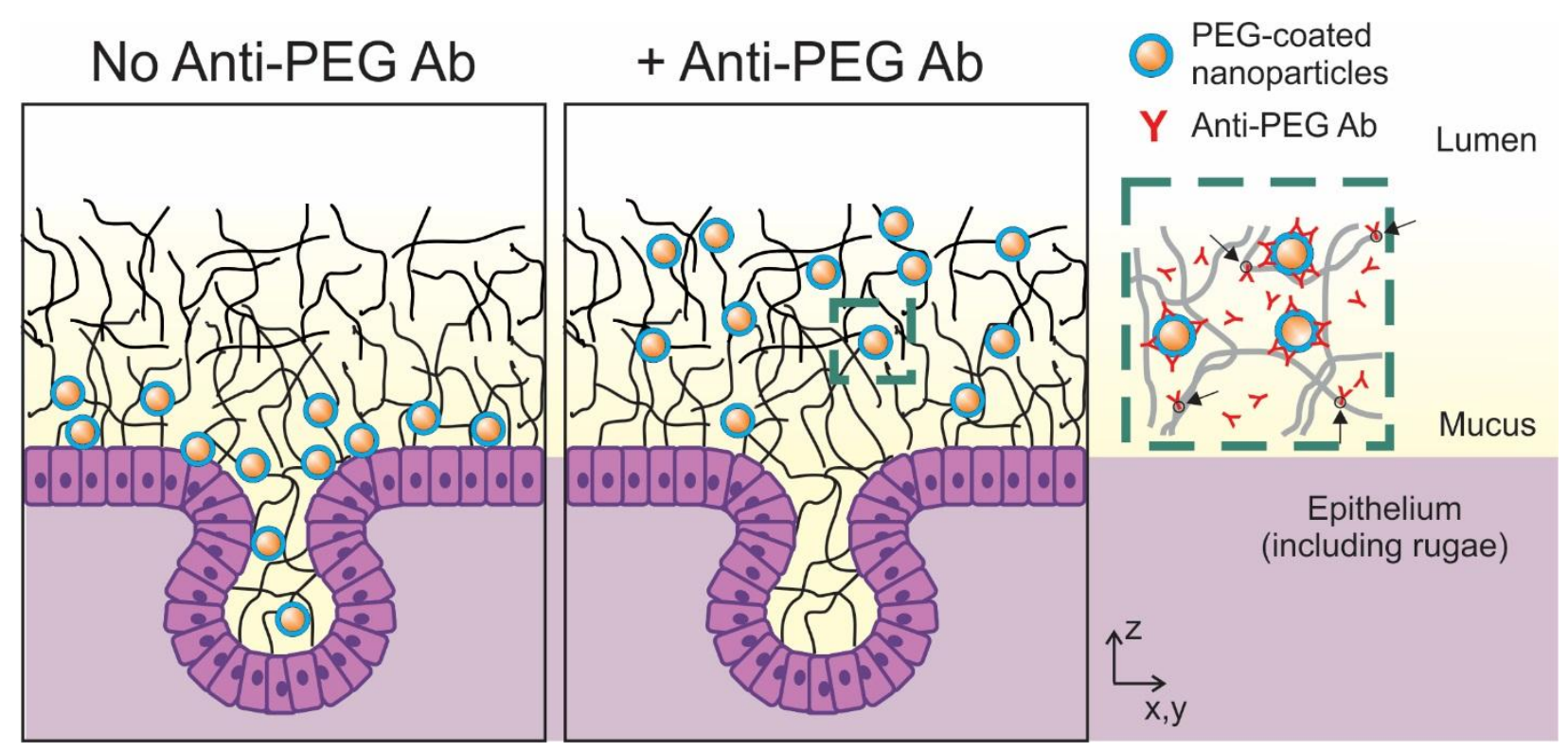




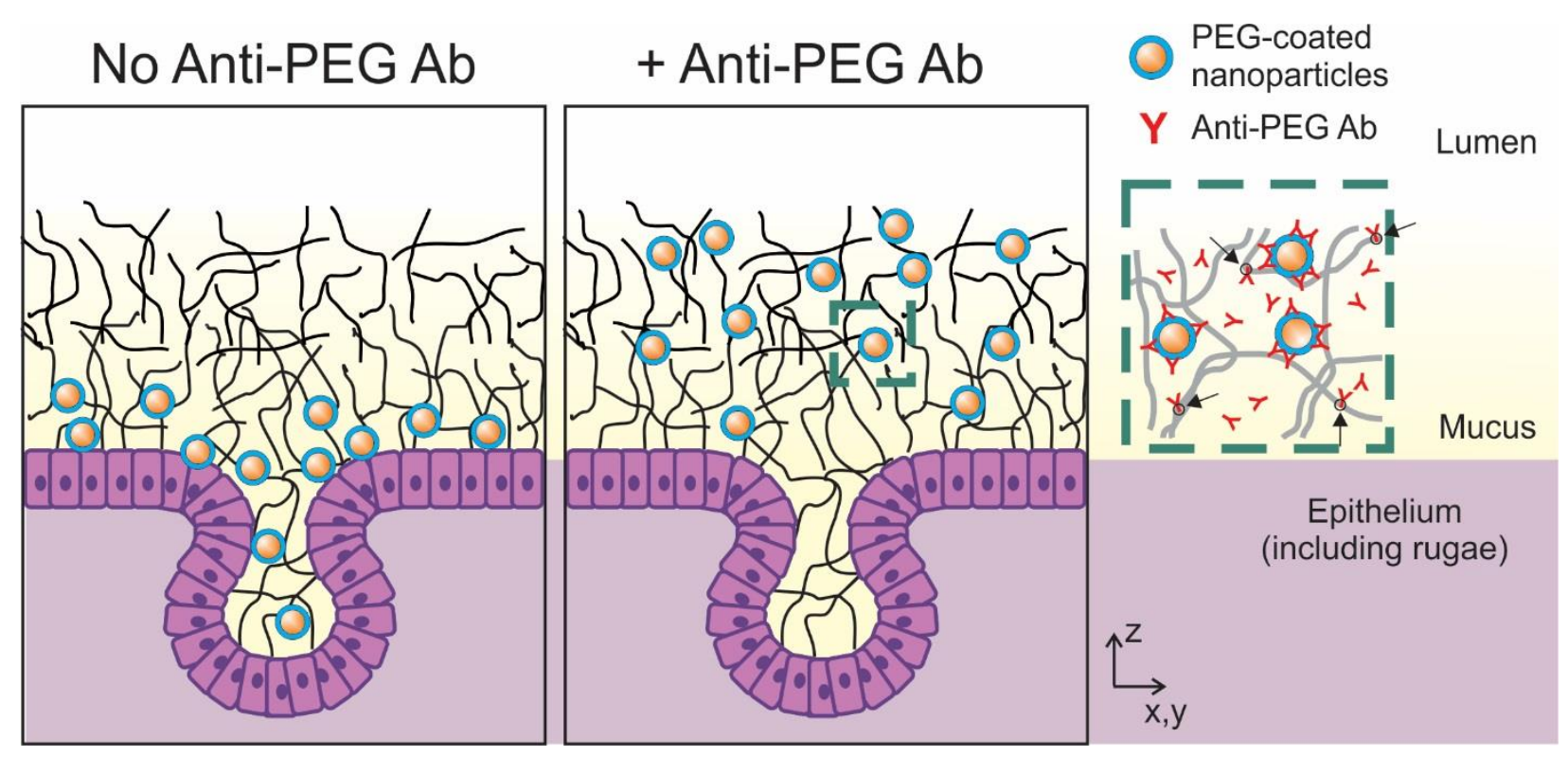

